\title{
Llamado a la acción de San Pablo para la prevención y el control de la hipertensión arterial, 2020
}

\author{
Norm RC Campbell', Aletta E Schutte ${ }^{2}$, Cherian V Varghese ${ }^{3}$, Pedro Ordunez ${ }^{4}$, Xin-Hua Zhang ${ }^{5}$, \\ Taskeen Khan ${ }^{3}$, James E Sharman ${ }^{6}$, Paul K Whelton ${ }^{7}$, Gianfranco Parati ${ }^{8}$, Michael A Weber ${ }^{9}$, \\ Marcelo Orías ${ }^{10}$, Marc G Jaffe ${ }^{11}$, Andrew E Moran ${ }^{12}$, Frida Liane Plavnik ${ }^{13}$, Venkata S Ram ${ }^{14}$, \\ Michael Brainin ${ }^{15}$, Mayowa O Owolabi ${ }^{16}$, Agustin J Ramirez ${ }^{17}$, Eduardo Barbosa ${ }^{18}$, \\ Luiz Aparecido Bortolotto ${ }^{19}$, Daniel T Lackland ${ }^{20}$
}

Forma de citar Campbell NRC, Schutte AE, Varghese CV, Ordunez P, Zhang XH, Khan T et al. Llamado a la acción de San Pablo para la prevención y el control de la hipertensión arterial, 2020. Rev Panam Salud Publica. 2021;45:e26. https://doi.org/10.26633/ RPSP.2021.26

RESUMEN

- Cerca de una cuarta parte de los adultos tienen hipertensión, el principal factor de riesgo de muerte (inclusive la causada por cardiopatía y accidente cerebrovascular).

- Existen políticas eficaces que podrían ayudar a las personas a elegir opciones saludables para prevenir el aumento de la presión arterial; si se las aplicara plenamente, se podría evitar en gran medida el desarrollo de hipertensión.

- La hipertensión es fácil de detectar y tratar, PERO solo alrededor de $50 \%$ de los adultos que presentan dicha afección son conscientes de su situación y solamente 1 de cada 7 de ellos recibe el tratamiento adecuado.

- La prevención y el control de la hipertensión es el mecanismo principal para prevenir y controlar las enfermedades no transmisibles y un modelo para evitar otros riesgos de presentar dichas enfermedades.

- La adopción de un modo de vida saludable y el tratamiento farmacológico efectivo podrían prevenir y controlar la hipertensión en la mayoría de las personas si se implementaran de manera sistemática en la población; en todos los entornos es posible aplicar intervenciones sencillas, que pueden usarse para mejorar la atención primaria

- Es urgente adoptar medidas sostenidas para introducir cambios eficaces en las políticas públicas y los sistemas de salud pública con miras a prevenir y controlar la hipertensión.

Palabras clave Hipertensión; enfermedades cardiovasculares; salud global; dieta hiposódica; accidente cerebrovascular.

\footnotetext{
* Traducción oficial al español del artículo original en inglés efectuada por la Organización Panamericana de la Salud, con autorización de Wiley Periodicals. En caso de discrepancia, prevalecerá la versión original en inglés. Wiley no se hace responsable de la exactitud de la traducción del original inglés ni de los eventuales errores que esta pueda contener.

Cita del artículo original: Campbell NRC, Schutte AE, Varghese CV, et al. São Paulo call to action for the prevention and control of high blood pressure: 2020 . J Clin Hypertens. 2019;21:1744-1752. https:// doi.org/10.1111/jch.13741

1 Departamento de Medicina, Fisiología y Farmacología y Ciencias de la Salud de la Comunidad, Instituto O'Brien para Salud Pública e Instituto Cardiovascular Libin de Alberta, Universidad de Calgary, Calgary, Alberta, Canadá $\square$ Norm Campbell, ncampbel@ucalgary.ca

2 Unidad para la Hipertensión y las Enfermedades Cardiovasculares, Equipo de Investigación de la Hipertensión en África (HART), Universidad Noroccidental, Potchefstroom, Sudáfrica

3 Departamento de Enfermedades no Transmisibles, Organización Mundial de la Salud, Ginebra, Suiza
}

\footnotetext{
4 Departamento de Enfermedades no Transmisibles y Salud Mental, Organización Panamericana de la Salud, Washington, D.C., Estados Unidos

5 Instituto de la Liga de Beijing contra la Hipertensión, Beijing, China

6 Instituto Menzies de Investigación Médica, Universidad de Tasmania, Hobart, Tasmania, Australia

7 Departamentos de Epidemiología y Medicina, Centro de Ciencias Médicas de la Universidad de Tulane, Nueva Orleans, Estados Unidos

8 Departamento de Medicina y Cirugía, Universidad de Milán-Bicocca, y Departamento de Ciencias Cardiovasculares, Neurales y Metabólicas, Instituto Auxológico Italiano, IRCCS, Hospital San Luca, Milán, Italia

9 División de Medicina Cardiovascular, Universidad Estatal de Nueva York, Centro Médico Downstate, Brooklyn, Nueva York, Estados Unidos

10 Sanatorio Allende, y Universidad Nacional de Córdoba, Córdoba, Argentina

11 Resolve to Save Lives, una iniciativa de Vital Strategies, Nueva York, Estados Unidos y Kaiser Permanente de California del Norte, San Francisco meridional, California, Estados Unidos
} 


\section{INTRODUCCIÓN}

\subsection{Prevalencia de la hipertensión arterial}

- En el 2010, entre 1130 y 1400 millones de personas tenían hipertensión (definida como > 140/90 mmHg. https:// www.who.int/news-room/fact-sheets/detail/hypertension consultado el 16 de junio del 2019). ${ }^{1}$

- La presión arterial aumenta con la edad en las sociedades industrializadas, de tal manera que se estima que 9 de cada 10 adultos que alcancen los 80 años de edad desarrollarán hipertensión².

- Aproximadamente 1 de cada 4 adultos tienen hipertensión ( $\geq 140 / 90 \mathrm{mmHg}$ ), incluido el $40 \%$ de los mayores de 25 años (https: / / www.who.int/news-room/fact-sheets/detail/ hypertension, consultado el 16 de junio del 2019; https:/ / www.who.int/news-room/fact-sheets/detail/hypertension, consultado el 16 de junio del 2019) ${ }^{1,3}$.

- Si se definiera la hipertensión empleando un valor $\geq 130 / 80$ $\mathrm{mmHg}$, su prevalencia aumentaría aproximadamente 1,5 veces hasta afectar a aproximadamente el $50 \%$ de los adultos. ${ }^{4,5}$

\subsection{Repercusiones de la hipertensión en la salud}

- Según el estudio sobre la carga mundial de enfermedad, el aumento de la presión arterial es el principal factor de riesgo mundial prevenible de muerte, y el tercer mayor factor de riesgo de discapacidad. ${ }^{6}$

- En el 2017, el aumento de la presión arterial causó unos 10,4 millones de muertes (casi 19\% del total de muertes) y 218 millones de años de vida ajustados por discapacidad (AVAD) $\left(8,7 \%\right.$ del total de AVAD) ${ }^{6}$

- Cerca del 70\% de las muertes relacionadas con el aumento de la presión arterial suceden en personas con una presión arterial de $>140 / 90 \mathrm{mmHg}$, y el restante $30 \%$ en personas con una presión arterial subóptima, por debajo de 140/90 mmHg. ${ }^{7}$

- El aumento de la presión arterial causa más de 50\% de las cardiopatías, los accidentes cerebrovasculares y la insuficiencia cardíaca. ${ }^{7,8}$

- La hipertensión es el factor de riesgo más importante de accidente cerebrovascular isquémico así como hemorrágico. 9,10

- La hipertensión causa más del 40\% de las muertes en personas diabéticas ${ }^{11} \mathrm{y}$ también es un importante factor de riesgo de muerte fetal y materna en el embarazo, de demencia y de insuficiencia renal (en particular en personas de ascendencia africana), y puede causar ceguera.,12,13,14

\subsection{Repercusiones en las personas que viven en países de ingresos bajos, medianos y altos}

- La hipertensión es un importante factor de riesgo de muerte en los países de ingresos bajos, medianos y altos. ${ }^{7,15}$

\footnotetext{
12 Control Mundial de la Hipertensión, Resolve to Save Lives, una iniciativa de Vital Strategies, Nueva York, Estados Unidos

13 Grupo de Hipertensión, Hospital Alemão Oswaldo Cruz; e Instituto del Corazón (InCor); y Sociedad Brasileña de Hipertensión, San Pablo, Brasil)

14 Escuela de Medicina Sudoeste de la Universidad de Texas, Estados Unidos; Colegio Médico y Hospital Apollo, Hyderabad, India; Facultad de Medicina y Ciencias de la Salud de la Universidad de Macquarie, Sydney, Australia; Oficina Regional de Asia Meridional de la Liga Mundial de la Hipertensión, Hyderabad, India

15 Universidad del Danubio, Krems, Austria
}

- Dos tercios de las personas que padecen hipertensión se encuentran en países de ingresos bajos y medianos, donde vive la mayoría de la población mundial (https://www. who.int/news-room/fact-sheets/detail/hypertension, consultado el 16 de junio del 2019). ${ }^{1,3}$

- El número de adultos con hipertensión aumentó de 594 millones en 1975 a una cifra situada entre 1130 y 1400 millones, con el mayor aumento localizado en los países de ingresos bajos y medianos (https://www.who.int/newsroom/fact-sheets/detail/hypertension, consultado el 16 de junio del 2019).

- La presión arterial sistólica parece estar aumentando con el transcurso del tiempo en Asia oriental y meridional, Oceanía y África subsahariana, mientras que está disminuyendo en otras regiones. ${ }^{15}$

- En los países de ingresos bajos y medianos, la población joven presenta cardiopatías y accidentes cerebrovasculares. ${ }^{1,3}$

\subsection{Repercusiones económicas de la hipertensión}

- Se estima que $10 \%$ del gasto mundial en atención de salud se relaciona directamente con el aumento de la presión arterial y sus complicaciones, como la cardiopatía isquémica, la insuficiencia cardíaca y el accidente cerebrovascular. ${ }^{16}$

- Casi 25\% del gasto en atención de salud en Europa oriental y Asia central corresponde a enfermedades relacionadas con la presión arterial. ${ }^{16}$

- En el plan de acción mundial sobre las enfermedades no transmisibles, se estima que el manejo clínico de los riesgos de enfermedades cardiovasculares que incluyen la hipertensión, tiene una rentabilidad de la inversión superior a US\$ 3 por cada US\$ $1 .{ }^{17}$

\section{FACTORES DETERMINANTES DE LA HIPERTENSIÓN}

\subsection{Los entornos poco saludables desempeñan un papel importante en el aumento de la presión arterial}

- Los entornos poco saludables tienen una influencia determinante en los estilos de vida y los comportamientos poco saludables.

- Existe una amplia variación nacional en la prevalencia de los riesgos de desarrollar hipertensión (por ejemplo, riesgos alimentarios, inactividad física, obesidad, consumo excesivo de alcohol) que pueden determinar en gran medida diferentes tasas de prevalencia de esta afección. ${ }^{6}$

- La alimentación poco saludable (excluyendo el impacto alimentario sobre la obesidad) contribuye a cerca de la mitad de los pacientes con hipertensión. ${ }^{18}$

- Cerca del 30\% de los casos de hipertensión se relaciona con un mayor consumo de sodio (sal), y cerca del $20 \%$ se

\footnotetext{
16 Centro de Excelencia de las Universidades Africanas de Investigación sobre las

17 Enfermedades no Transmisibles, Universidad de Ibadan, Nigeria versitario, Fundación Favaloro, Buenos Aires, Argentina

18 Sociedad Latinoamericana de la Hipertensión, Artery Latam, Porto Alegre, Brasil

19 Hospital de Clínicas, Universidad de San Pablo; Instituto del Corazón; y Sociedad Brasileña de Hipertensión, San Pablo, Brasil

20 División de Neurociencias Aplicadas y Estudios de Población, Universidad Médica de Carolina del Sur, Charleston, Carolina del Sur, Estados Unidos
} 
relaciona con un aporte alimentario bajo de potasio (baja ingesta de frutas y hortalizas). ${ }^{18,19}$

- La elevada proporción de grasas saturadas frente a ácidos grasos no saturados también contribuye a la hipertensión. ${ }^{20}$

- La inactividad física se relaciona con aproximadamente el $20 \%$ de los casos de hipertensión. ${ }^{18}$

- La obesidad está asociada con cerca del 30\% de los casos de hipertensión. ${ }^{18}$

- El consumo excesivo de alcohol también aumenta la presión arterial y causa hipertensión. ${ }^{18}$

- No fumar es especialmente importante para que las personas con hipertensión prevengan enfermedades cardiovasculares y otras enfermedades no transmisibles. ${ }^{3}$

- Las desigualdades en cuanto a la educación y la situación socioeconómica repercuten significativamente en la prevalencia y el control de la hipertensión. ${ }^{21-23}$

- Los gobiernos han respaldado nueve metas mundiales voluntarias con el objetivo general de reducir en $25 \%$ la muerte prematura por las cuatro enfermedades no transmisibles principales para el 2025. Las metas relacionadas con la hipertensión y los factores de riesgo incluyen reducir en $25 \%$ la presión arterial no controlada, en 30\% el consumo de sodio alimentario, en 10\% la actividad física insuficiente, en 30\% el consumo de tabaco en las personas de 15 años o mayores, $y$ en detener el aumento de la obesidad y la diabetes. ${ }^{24}$

\subsection{En la mayoría de los países no se han aplicado sistemáticamente intervenciones clínicas para controlar la hipertensión}

- En todo el mundo, cerca de la mitad de los adultos con hipertensión desconocen que su presión arterial es alta, una situación que reviste mayor gravedad en los países de ingresos bajos. ${ }^{1,25}$

- Algunas de las personas que conocen que su presión arterial es alta siguen sin recibir tratamiento. Aun cuando estas reciben tratamiento, la mayoría alcanza un control subóptimo de la presión arterial. ${ }^{1}$

- En los países de ingresos altos las tasas promedio de concientización, tratamiento y control son de $67 \%$, 55,6\% y 28,4\% respectivamente, mientras que en los países de ingresos bajos y medianos son de $37,9 \%, 29 \%$ y 7,7\% respectivamente. ${ }^{1,26}$

- Menos de 1 de cada 5 adultos con hipertensión están controlados a nivel mundial y menos de 1 de cada 14 en África subsahariana (https:/ /www.who.int/news-room/fact-sheets/ detail/hypertension, consultado el 16 de junio del 2019) ${ }^{27}$.

\section{3. ¿QUÉ FUNCIONA?}

\subsection{Las inversiones en prevención a menudo reducen los costos}

- Las políticas de intervenciones a nivel poblacional para crear entornos que mejoren la alimentación y la actividad física suelen reducir los costos y ayudan a que las personas elijan opciones saludables. $^{28,29}$

- Se estima que la reducción del sodio alimentario tiene un rendimiento de la inversión de US\$ 13 a US\$ 18 por cada dólar invertido. ${ }^{17,30}$

- Las políticas recomendadas para prevenir o controlar las enfermedades no transmisibles, incluida la hipertensión, consisten en mejorar la alimentación, aumentar la actividad física, evitar el consumo de tabaco y reducir el consumo nocivo de alcohol, y están definidas por la Organización Mundial de la Salud. ${ }^{31,32,33}$ (https://www.who.int/tobacco/ mpower/publications/en/, consultado el 7 de mayo del 2019).

\subsection{Las inversiones en el tratamiento y control son eficaces en función de los costos}

- El tratamiento eficaz de la presión arterial de > 140/90 $\mathrm{mmHg}$ reduce el accidente cerebrovascular y la cardiopatía. ${ }^{34}$

- El tratamiento eficaz de la presión arterial de > 130/80 $\mathrm{mmHg}$ reduce el accidente cerebrovascular y la cardiopatía en las personas con riesgo cardiovascular moderado a alto (riesgo de $>10-19 \%$ de sufrir enfermedades cardiovasculares en los próximos 10 años en personas con riesgo moderado y $>20 \%$ de sufrir enfermedades cardiovasculares en los próximos 10 años en personas con riesgo elevado) ${ }^{34}$.

- En los Estados Unidos, Canadá y Finlandia, más del $80 \%$ de las personas con hipertensión presentan otros riesgos cardiovasculares o evidencia de daños relacionados con la presión arterial (cardiopatía, accidente cerebrovascular o daño renal) ${ }^{35,36}$.

- La evaluación objetiva del riesgo cardiovascular absoluto puede ser útil en el manejo general de los pacientes, incluso en la necesidad potencial de emplear un umbral de tratamiento y un objetivo terapéutico más bajos, así como en el tratamiento de la dislipidemia. ${ }^{34,37-39}$

- El tratamiento de la hipertensión es la piedra angular rutinaria de la prevención primaria de las enfermedades no transmisibles y proporciona un fundamento para realizar el tamizaje periódico de la presión arterial en todos los adultos y tratar a los que presentan hipertensión.

- Los resultados del estudio SPRINT y un nuevo análisis del estudio ACCORD enfatizan que, en general, el tratamiento intensivo de la presión arterial es beneficioso para reducir la mortalidad en las personas con riesgo elevado de sufrir enfermedad cardiovascular, aunque puede causar otros efectos colaterales. ${ }^{40,41}$

\section{4. ¿CUÁLES SON LOS OBSTÁCULOS?}

\subsection{La inercia en materia de políticas}

- Muchos países no han puesto en práctica políticas públicas eficaces de amplio alcance que propicien una alimentación y una actividad física óptimas, el control del consumo de alcohol y tabaco, y la prevención de muchas enfermedades no transmisibles, incluidos los riesgos como la hipertensión. ${ }^{33}$ (https://www.wcrf.org/int/policy/nourishing-database, consultado el 17 de abril del 2019; https://www.who. int/news-room/fact-sheets/detail/physical-activity, consultado el 5 de mayo del 2019; \%https://www.who.int/ substance_abuse/activities/fadab/msb_adab_gas_progress_ report.pdf?ua=1, consultado del 5 de mayo del 2019 ).

- Muchos países no han ampliado a mayor escala la atención primaria para atender las necesidades de salud de la población, incluidos el diagnóstico y el tratamiento de la hipertensión. ${ }^{42,43}$

- Muchas organizaciones y sociedades nacionales de lucha contra la hipertensión y las enfermedades cardiovasculares 
no tienen planes estratégicos para prevenir, diagnosticar, tratar y controlar la hipertensión, y no impulsan eficazmente las políticas que estén en consonancia con las que ha elaborado la OMS para la prevención y el control eficaces de las enfermedades no transmisibles, incluida la hipertensión.

\subsection{La inercia de los sistemas de salud}

- Aunque mejorar el control de la hipertensión es una de las intervenciones clínicas más eficaces en función de los costos para reducir la carga de enfermedades no transmisibles y alcanzar los objetivos de desarrollo sostenibles (ODS), en muchos países la prevención y el control de la hipertensión no son una alta prioridad política y sanitaria. ${ }^{44}$ La mayoría de los países no tienen un enfoque estratégico de los sistemas de salud y la salud pública para controlar la hipertensión. ${ }^{45}$

- Un enfoque estratégico de los sistemas de salud y la salud pública para controlar la hipertensión incluye la cobertura de toda la población por dichos sistemas; el acceso fácil a un suministro fiable y asequible de una serie de medicamentos antihipertensivos de alta calidad, incluidas las combinaciones de una sola pastilla; una atención de fácil acceso basada en el trabajo en equipo; la medición o evaluación sistemática de presión arterial en todas las consultas clínicas; el tamizaje comunitario de la hipertensión estrechamente vinculado con el sistema de salud; el uso por personal recientemente capacitado de monitores electrónicos de la presión arterial validados; el uso de un algoritmo normativo sencillo de diagnóstico y tratamiento; el uso de un registro de la hipertensión con informes de resultados; y encuestas periódicas (cada 3 a 5 años) sobre la hipertensión en la población. ${ }^{46-52}$

- Muchos médicos no inician o valoran adecuadamente el tratamiento en las personas que presentan valores elevados de presión arterial. ${ }^{53,54}$

\section{AGENDA DE TRANSFORMACIÓN PARA LA PREVENCIÓNY EL CONTROL DE LA HIPERTENSIÓN}

- Las políticas e intervenciones transformadoras de los servicios de salud pueden contribuir a mejorar los servicios de atención primaria de salud y el control de la hipertensión, y son altamente recomendadas por la Organización Mundial de la Salud, la estrategia Resolve to Save Lives, la Sociedad Internacional de la Hipertensión, la Liga Mundial de la Hipertensión y otras organizaciones.

- La Organización Mundial de la Salud ha estado al frente de múltiples iniciativas con organizaciones internacionales en el desarrollo de recursos de última generación que definan las políticas e intervenciones para prevenir y controlar las enfermedades cardiovasculares y la diabetes. El recurso más destacado y actualizado es el paquete técnico HEARTS (https:/ / www.who.int/cardiovascular_diseases/hearts / en/, consultado el 17 de abril del 2019).

- Resolve to Save Lives (RTSL), una iniciativa de la organización Vital Strategies, es un programa mundial financiado con apoyo filantrópico destinado a mejorar el control de la hipertensión, reducir el consumo alimentario de sodio, eliminar las grasas trans artificiales de la alimentación y mejorar la preparación para una pandemia (https://www.resolvetosavelives.org/, consultado el 17 de abril del 2019).
- A fin de controlar la hipertensión, el paquete técnico HEARTS aboga firmemente por aumentar la capacidad clínica de la atención primaria basada en el trabajo de equipo, el suministro confiable y asequible de medicamentos antihipertensivos eficaces (incluidos las combinaciones de una sola pastilla cuando sea apropiado), la medición o evaluación sistemática de la presión arterial en todas las consultas clínicas, el uso por personal recientemente capacitado de monitores electrónicos de la presión arterial validados, el uso de un algoritmo normativo sencillo para el diagnóstico y el tratamiento, el uso de un registro de la hipertensión con informes de resultados, y el uso de encuestas periódicas sobre la hipertensión en la población.

- Todos los años la Sociedad Internacional de la Hipertensión organiza junto con diversas organizaciones asociadas el Mes de la Medición de la presión arterial, y cada mes de mayo se llevan a cabo actividades de detección de la presión arterial usando técnicas estandarizadas para crear una mayor conciencia sobre el aumento de la hipertensión. A su vez, la Liga Mundial de la Hipertensión organiza anualmente con organizaciones asociadas el Día Mundial de la Hipertensión (17 de mayo) para aumentar la concientización sobre la hipertensión. $25,55-57$

\section{UN ENFOQUE CLÍNICO TRANSFORMADOR}

\subsection{Profesionales de la salud y establecimientos y organizaciones de atención de salud}

- Mantenerse al día de las mejores normas mundiales de prevención y control de la hipertensión.

- Medir la presión arterial en todas las consultas clínicas apropiadas.

- Adquirir dispositivos automatizados de medición de la presión arterial que hayan sido validados para medir con exactitud la presión arterial, y promover e imponer su uso.

- Elaborar programas estandarizados de capacitación sobre la medición exacta de la presión arterial para pacientes y profesionales de la salud.

- Asegurarse de que el personal que mide la presión arterial haya sido capacitado y evaluado de forma periódica para garantizar las lecturas precisas de la presión arterial. Exigir cursos de certificación donde estos estén disponibles.

- Evaluar el riesgo de enfermedad cardiovascular usando un método objetivo (programa informático, cuadro de riesgos), y determinar la presencia de enfermedad cardiovascular o de hipertensión relacionados con daño de órgano blanco como, por ejemplo, la enfermedad renal crónica en las personas diagnosticadas con hipertensión. Abordar todos los riesgos detectados de enfermedad cardiovascular con las pautas nacional o internacionalmente reconocidas. $.88,59$

- La atención basada en el trabajo de equipo mejora el tratamiento y el control de la hipertensión. ${ }^{34,60}$ Optimizar la atención centrada en el paciente por medio de un enfoque basado en el trabajo de equipo para medir la presión arterial, establecer contacto periódico con el paciente y llevar a cabo otras tareas ordinarias dentro del alcance normativo de la práctica. Además de usar un enfoque basado en el trabajo de equipo, con profesionales no médicos (personal administrativo del consultorio, enfermeras, farmacéuticos, agentes de 
salud comunitarios, etc.) ejerciendo al máximo de su certificación o facultades, el personal de oficina, los cónyuges y los amigos pueden desempeñar un papel importante para mejorar la efectividad del tratamiento. Sin embargo, el paciente siempre debe permanecer en el centro del equipo.

- Evaluar en cada paciente la conveniencia de que sea diagnosticado y tratado usando un algoritmo o protocolo sencillo de manejo de la hipertensión y personalizar el tratamiento para la pequeña proporción de pacientes inadecuados para el algoritmo o que sufren efectos colaterales o intolerancia al medicamento. ${ }^{61}$

- Tratar inmediatamente a las personas con una presión arterial mayor o igual a 160/100 mmHg después de haber hecho el diagnóstico, colocar tratamiento antihipertensivo y recomendar cambios en el estilo de vida.

- Exigir el uso de medicamentos antihipertensivos de alta calidad haciendo hincapié en aquellos que cumplen normas de calidad aceptables, tienen una acción prolongada, pueden usarse una vez al día y están constantemente disponibles para evitar la confusión y la interrupción del tratamiento cuando se cambian los medicamentos (aun cuando sean clínicamente equivalentes).

- Evaluar periódicamente el cumplimiento del paciente del esquema terapéutico para el tratamiento de la hipertensión.

- Usar registros con informes de resultados.

- Evaluar los hábitos alimentarios, el consumo de tabaco, el consumo de alcohol, la actividad física y la obesidad, y prestar asesoramiento individualizado sobre el modo de vida. Informar y ayudar específicamente a todos los consumidores de tabaco a dejar de fumar, e informar y ayudar a todos los consumidores de alcohol en grandes cantidades a limitar dicho consumo o abandonarlo.

- Promover y prestar asistencia a los programas comunitarios de detección de la presión arterial, como el Mes de mayo para la Medición de la Presión arterial y el Día Mundial de la Hipertensión (17 de mayo).

- Educar a los pacientes, sus familiares y el público.

\subsection{Atención primaria}

- Asegurarse de que las organizaciones que representan a los prestadores de atención primaria de salud que previenen, diagnostican y tratan la hipertensión sean fundamentales en las estrategias para controlar la hipertensión.

- Asegurarse con las organizaciones de atención primaria de que estén disponibles directrices terapéuticas para la hipertensión adaptadas a la población del país. Traducir las directrices en algoritmos o protocolos de diagnóstico y tratamiento que sean sencillos y fáciles de usar, e incluyan combinaciones de medicamentos de una sola pastilla. ${ }^{46,61,62}$

- Elaborar programas estandarizados de educación con las organizaciones de atención primaria que se centren en las intervenciones claves para tratar y controlar sistemáticamente la hipertensión en la atención primaria. ${ }^{63}$

- Donde sea factible, usar los avances tecnológicos (sistemas de salud digital y por telefonía móvil) para involucrar y empoderar a los pacientes. ${ }^{64-66}$

- Establecer sistemas de medición domiciliaria de la presión arterial combinados con el uso de telemetría para transmitir los valores registrados en el domicilio a los profesionales de la salud y solicitarles asesoramiento con objeto de optimizar el tratamiento, mejorar la adhesión de los pacientes y reducir la inercia terapéutica.

- Los teléfonos inteligentes con aplicaciones relacionadas con la gestión de la hipertensión pueden proporcionar recursos de telemedicina de bajo costo para la práctica diaria. Una de estas aplicaciones (ESH CARE) de la Sociedad Europea de Hipertensión ha sido validada y se actualiza continuamente. . $5,67-69^{6}$

\subsection{Reconocimientos y premios}

- Postular a los programas, los representantes de programas y las personas individuales que lo merezcan a los premios de reconocimiento de la Liga Mundial de la Hipertensión y la Sociedad Internacional para la Hipertensión por su labor en prodela reducción delconsumodesal por la población yelcontrol de la presión arterial (http: / / www.whleague.org/index. php/news-awards-recognition, consultado el 17 de abril del 2019; http://ish-world.com/activities/awards-prizes. htm, consultado el 5 de mayo del 2019).

\subsection{Promoción de la causa}

- Abogar por políticas públicas favorables a la salud, especialmente las que reducen el consumo de sal o sodio alimentarios, promueven una alimentación saludable y la actividad física, reducen el consumo nocivo de alcohol y eliminan el consumo de tabaco y de las grasas trans artificiales. ${ }^{31}$

- Impulsar o fomentar la actualización de los formularios terapéuticos nacionales para asegurar la disponibilidad y asequibilidad de los medicamentos antihipertensivos de alta calidad haciendo hincapié en los fármacos de acción prolongada y la combinación de medicamentos de una sola pastilla.

- Abogar por políticas que incluyan la regulación para garantizar la compra, la venta y el uso de dispositivos automatizados y manguitos para medir la presión arterial cuya exactitud e idoneidad hayan sido validadas. ${ }^{70,71}$

- Abogar por la capacitación periódica y la certificación de los profesionales de la salud y demás personas que miden regularmente la presión arterial. ${ }^{72}$

- Impulsar la atención primaria colaborativa basada en el trabajo de equipo y centrada en el paciente. ${ }^{50}$

- Promover el uso de registros de hipertensión para el seguimiento clínico y la retroalimentación sobre el desempeño en los consultorios que atienden a personas con hipertensión. 49,73,74

- Fomentar las iniciativas estandarizadas de seguimiento y evaluación para prevenir y controlar la hipertensión. . $9,74-76$

- Supervisar los informes nacionales periódicos (trimestrales o anuales) sobre las tasas de control de la hipertensión, y apoyar y promover los esfuerzos para mejorarlas.

\subsection{Personas empoderadas}

- Basar la alimentación principalmente en una variedad de verduras (especialmente de hojas verdes), frutas, frutos secos, semillas y legumbres. ${ }^{77}$

- Comer con mayor frecuencia alimentos sin procesar o mínimamente procesados.

- Elegir las opciones bajas de sodio y no agregar sal a los alimentos.

- No consumir tabaco.

- Ser físicamente activas. 
- Alcanzar y mantener un peso corporal saludable.

- Limitar el consumo de bebidas alcohólicas y evitar exceder las recomendaciones de consumo máximo diario y semanal de alcohol.

- Controlar la presión arterial regularmente y saber qué valor debería tener.

- Medir la propia presión arterial. Aprender a obtener mediciones exactas de la presión arterial y saber interpretarlas, en colaboración con el equipo de atención de salud.

- Abogar por políticas públicas favorables a la salud.

- Exigir el uso de medicamentos antihipertensivos de alta calidad haciendo hincapié en los que sean de acción prolongada.

- Estar en contacto con su prestador de atención de salud por medio de herramientas de salud digital y de telefonía móvil validadas.

- Cuando se receten medicamentos para el tratamiento de la hipertensión, tomarlos regularmente hasta que sean cambiados por un profesional de la salud.

- Establecer una rutina para la medicación prescrita y supervisar la toma de los medicamentos para lograr el cumplimiento terapéutico.

\section{RECURSOS MUNDIALES SOBRE LAS MEJORES PRÁCTICAS}

- Los recursos de la OMS y RTSL en materia de enfermedades no transmisibles para mejorar el control de la hipertensión pueden consultarse en el Portal conocimientos y acción (https:/ / www.knowledge-action-portal.com/ consultado el 17 de abril del 2019), en LINKS, una comunidad mundial para la salud cardiovascular (https:/ / www.linkscommunity. org/ consultado el 17 de abril del 2019) y en https:/ /www. who.int/cardiovascular_diseases/hearts/en/, (consultado el 3 de junio del 2019).

- Los recursos educativos y de capacitación en materia de prevención y tratamiento de la hipertensión también están disponibles en el sitio web de la Organización Panamericana de la Salud (https://www.paho.org/ hq $/$ index.php?option $=$ com_content $\& v i e w=$ article \&i$\mathrm{d}=15056$ : hearts-in-the-americas\&Itemid $=3465 \&$ lang $=$ en, consultado el 3 de junio del 2019) y en el Campus Virtual de Salud Pública (https://mooc.campusvirtualsp.org/enrol/ index.php?id=35, consultado el 6 de mayo del 2019).

- Los recursos de la Liga Mundial de la Hipertensión pueden encontrarse en http:/ /www.whleague.org/ (consultado el 6 de mayo del 2019).

- Los recursos de la Sociedad Internacional para la Hipertensión pueden encontrarse en http://ish-world.com/index. htm (consultado el 6 de mayo del 2019).

La presente nota descriptiva y llamado a la acción cuenta con el apoyo de la Liga Mundial de la Hipertensión, la Sociedad Argentina para la Hipertensión, la Oficina Regional de Asia-Pacífico de la Liga Mundial de la Hipertensión, la Oficina Regional del Sur de Asia de la Liga Mundial de la Hipertensión, la Alianza Universitaria Africana de Investigación, el Comité Belga de la Hipertensión, Blue Cross y Blue Shield de Louisiana, la Sociedad Brasileña para la Hipertensión, la Liga Búlgara de la Hipertensión, la Caja Costarricense de Seguro Social, la Fundación para el Corazón de Camerún, la Liga China de la Hipertensión,
Colleagues in Care, Hypertension Canada, la Sociedad Europea para la Hipertensión, el Consejo para la Hipertensión de la Sociedad Europea de Cardiología, el Centro de Excelencia Ibadan para las Enfermedades No Transmisibles, la Sociedad India de Hipertensión, el Consejo Internacional de Prevención y Rehabilitación Cardiovascular, la Sociedad Internacional para la Hipertensión, la Sociedad Internacional para el Estudio de la Hipertensión Gestacional, la Sociedad Internacional de Nefrología,la fundación Iraní para el Corazón, el Grupo de la Comisión Lancet para la Hipertensión, la Sociedad Latinoamericana de Nefrología e Hipertensión, la Sociedad Letona para la Hipertensión y la Aterosclerosis, la Sociedad para la Hipertensión de Mongolia, el Comité Técnico Consultivo del Programa de Hipertensión Arterial de Cuba (Ministerio de Salud Pública), el Centro de Excelencia NHMRC de Investigación sobre el Mejoramiento de Resultados Cardiovasculares (Australia), la Fundación Onom (Mongolia), la Sociedad Filipina de Hipertensión, Resolve to Save Lives, la Sociedad Saudí para el Manejo de la Hipertensión, la Sociedad Portuguesa para la Hipertensión, la Oficina Regional de Asia Meridional de la Liga Mundial de la Hipertensión, la iniciativa Stroke Control Innovations de Nigeria, Stroke Investigative Research and Educational Network (SIREN), la Sociedad Sudanesa de Hipertensión, la Sociedad Sueca de Hipertensión, Stroke and Vascular Medicine, la Red de Apoyo de la Asociación de Accidente Cerebrovascular de Ghana, la Sociedad de Taiwán para la Hipertensión, la Sociedad de Taiwán de Cardiología, la Sociedad Tailandesa de Hipertensión, la Escuela de Enfermería de la Universidad de San Pablo en Ribeirão Preto, el Grupo de Acción Mundial sobre la Hipertensión, la Cumbre Mundial de Salud, y World Stroke Organization.

Conflicto de intereses. NRCC fue consultor asalariado de la Fundación Novartis (2016-2017) en apoyo de su programa de mejora del control de la hipertensión en los países de ingresos bajos y medianos, lo que incluyó recibir fondos de apoyo para viajes a visitas in situ y un contrato para llevar a cabo una encuesta. NRCC prestó asesoramiento consultivo remunerado sobre la medición exacta de la presión arterial para Midway Corporation (2017) y es miembro no remunerado de la Acción Mundial sobre Sal y Salud (WASH). AES recibió honorarios de oradora de Novartis y Omron para realizar conferencias científicas sobre la presión arterial y la evaluación de riesgos, y de Servier para disertar sobre la concientización acerca de la medición de la presión arterial. Es consultora asalariada de Abbott Pharmaceuticals sobre la medicación antihipertensiva y preside la Sociedad Internacional para la Hipertensión. MAW trabajó como consultor para Abbvie y Bristol Myers Squibb sobre la inocuidad de los medicamentos y recibió fondos para viajes de Omron. Es miembro de las juntas asesoras de Medtronic, ReCor y Ablative Solutions. MO declara haber recibido honorarios de Glaxo Smith Kline (GSK) y Takeda Inc. para celebrar conferencias. GP informa haber percibido honorarios de Omron Health Care, Sanofi y Servier. AR comunica haber prestado apoyo en conferencias para Servier. PW, LAB, PO, XHZ, CV, TK, DL, MJ, AM, FLP, MB, EB y JS no tienen conflictos de intereses financieros para declarar.

Declaración. Las opiniones, resultados y conclusiones expresados en este manuscrito son únicamente responsabilidad de los autores y no reflejan necesariamente los criterios ni la política de la RPSP, la Organización Panamericana de la Salud o la Organización Mundial de la Salud. 


\section{REFERENCIAS}

1. Mills KT, Bundy JD, Kelly TN, et al. Global Disparities of Hypertension Prevalence and Control: A Systematic Analysis of Population-Based Studies From 90 Countries. Circulation. 2016;134(6):441-450.

2. Vasan RS, Beiser A, Seshadri S, et al. R Residual Lifetime Risk for Developing Hypertension in Middle-aged Women and Men. JAMA. 2002;287(8):1003-1010.

3. Organización Mundial de la Salud. Información general sobre la hipertensión en el mundo: Una enfermedad que mata en silencio, una crisis de salud pública mundial. Día Mundial de la Salud 2013. Ginebra: OMS;2013.

4. Muntner P, Carey RM, Gidding S, et al. Potential U.S. Population Impact of the 2017 American College of Cardiology/American Heart Association High Blood Pressure Guideline. J Am Coll Cardiol. 2018;71(2):109-118.

5. Wang JG, Liu L. Global Impact of 2017 American College of Cardiology/American Heart Association Hypertension Guidelines: A Perspective From China. Circulation. 2018;137(6):546-548.

6. Global Burden of Disease (sitio web). Instituto de Sanimetría y Evaluación Sanitaria. http://vizhub.healthdata.org/gbd-compare/. Consultado el 13 de julio del 2019.

7. Forouzanfar MH, Liu P, Roth GA, et al. Global Burden of Hypertension and Systolic Blood Pressure of at Least 110 to $115 \mathrm{~mm} \mathrm{Hg}$, 1990-2015. JAMA. 2017;317(2):165-182.

8. Levy D, Larson MG, Vasan RS, Kannel WB, Ho KKL. The Progression From Hypertension to Congestive Heart Failure. JAMA. 1996;275(20):1557-1562.

9. Feigin VL, Roth GA, Naghavi M, et al. Global burden of stroke and risk factors in 188 countries, during 1990\&\#x2013;2013: a systematic analysis for the Global Burden of Disease Study 2013. Lancet Neurol. 2016;15(9):913-924.

10. O'Donnell MJ, Chin SL, Rangarajan S, et al. Global and regional effects of potentially modifiable risk factors associated with acute stroke in 32 countries (INTERSTROKE): a case-control study. Lancet. 2016;388(10046):761-775.

11. Chen G, McAlister FA, Walker RL, Hemmelgarn BR, Campbell NR. Cardiovascular outcomes in framingham participants with diabetes: the importance of blood pressure. Hypertension. 2011;57(5):891-897.

12. Khan KS, Wojdyla D, Say L, Gulmezoglu AM, Van Look PFA. WHO analysis of causes of maternal death: a systematic review. Lancet. 2006;367(9516):1066-1074.

13. Levi MN, Macquin-Mavier I, Tropeano AI, Bachoud-Levi AC, Maison P. Antihypertensive classes, cognitive decline and incidence of dementia: a network meta-analysis. J Hypertens. 2013;31(6):1073-1082.

14. Udani S, Lazich I, Bakris GL. Epidemiology of hypertensive kidney disease. Nat Rev Nephrol. 2011;7(1):11-21.

15. Worldwide trends in blood pressure from 1975 to 2015: a pooled analysis of 1479 population-based measurement studies with 19.1 million participant. Lancet. 2017;389(10064):37-55.

16. Gaziano TA, Bitton A, Anand S, Weinstein MC. The global cost of nonoptimal blood pressure. J Hypertens. 2009;27(7):1472-1477.

17. Organización Mundial de la Salud. Salvar vidas, reducir el gasto: Una respuesta estratégica a las enfermedades no transmisibles. Ginebra: OMS; 2018 (OMS/NMH/NVI/18.8). Licencia: CC BY-NC-SA 3.0 IGO.

18. Institute of Medicine of the National Academies. A Population-Based Policy and Systems Change Approach to Prevent and Control HypertensionBrief Report. Washington, D.C.: National Academy Press; 2010.

19. Joffres M, Campbell NR, Manns B, Tu K. Estimate of the benefits of a population-based reduction in dietary sodium additives on hypertension and its related health care costs in Canada. Can J Cardiol. 2007;23(6):437-443.

20. Puska P, Iacono JM, Nissinen A, et al. Dietary fat and blood pressure: an intervention study on the effects of a low-fat diet with two levels of polyunsaturated fat. Prev Med. 1985;14(5):573-584.

21. Leng B, Jin Y, Li G, Chen L, Jin N. Socioeconomic status and hypertension: a meta-analysis. J Hypertens. 2015;33(2):221-229.

22. Chor D, Pinho Ribeiro AL, Sa Carvalho M, et al. Prevalence, Awareness, Treatment and Influence of Socioeconomic Variables on Control of High Blood Pressure: Results of the ELSA-Brasil Study. PloS ONE. 2015;10(6):e0127382.
23. Liew SJ, Lee JT, Tan CS, Koh CHG, Van Dam R, MullerRiemenschneider F. Sociodemographic factors in relation to hypertension prevalence, awareness, treatment and control in a multi-ethnic Asian population: a cross-sectional study. BMJ open. 2019;9(5):e025869.

24. Organización Mundial de la Salud. Global status report on noncommunicable disease 2014. Ginebra: OMS; 2014. 9789241564854.

25. Beaney T, Burrell LM, Castillo RR, et al. May Measurement Month 2018: a pragmatic global screening campaign to raise awareness of blood pressure by the International Society of Hypertension. Eur Heart J. 2019; doi:10.1093/eurheartj/ehz300.

26. Torlasco C, Faini A, Makil E, et al. Cardiovascular risk and hypertension control in Italy. Data from the 2015 World Hypertension Day. Int J Cardiol. 2017;243:529-532.

27. Ataklte F, Erqou S, Kaptoge S, Taye B, Echouffo-Tcheugui JB, Kengne AP. Burden of Undiagnosed Hypertension in Sub-Saharan Africa: A Systematic Review and Meta-Analysis. Hypertension. 2015:291-298.

28. Masters R, Anwar E, Collins B, Cookson R, Capewell S. Return on investment of public health interventions: a systematic review. J Epidemiol Community Health. 2017;71(8):827-834.

29. Foro Económico Mundial, Escuela de Salud Pública de Harvard, Organización Mundial de la Salud. From Burden to "Best Buys": Reducing the Economic Impact of Non-Communicable Diseases in Low- and Middle-Income Countries. Ginebra: Foro Económico Mundial;2011.

30. Nugent R. Benefits and Costs of the Non-Communicable Disease Targets for the Post-2015 Development Agenda. Copenhagen Consensus Center. 2015:I-II, 1-25.

31. Organización Mundial de la Salud. Plan de acción mundial de la OMS para la prevención y control de enfermedades no transmisibles 2013-2020. Ginebra: OMS; 2013.

32. Organización Panamericana de la Salud. SHAKE: Menos sal, más salud. Guía técnica para reducir el consumo de sal. Washington, DC: OPS, 2018.

33. Organización Mundial de la Salud. Estrategia mundial para reducir el uso nocivo del alcohol. Ginebra: OMS; 2010.

34. Whelton PK, Carey RM, Aronow WS, et al. ACC/AHA/AAPA/ ABC/ACPM/AGS/APhA/ASH/ASPC/NMA/PCNA Guideline for the Prevention, Detection, Evaluation, and Management of High Blood Pressure in Adults: A Report of the American College of Cardiology/American Heart Association Task Force on Clinical Practice Guidelines. Hypertension. 2017;71:e13-e115.

35. McAlister FA, Robitaille C, Gillespie C, et al. The Impact of Cardiovascular Risk Factor Profiles on Blood Pressure Control Rates in Adults From Canada and the United States. Can J Cardiol. 2013;29:598-605.

36. Rantala AO, Kauma H, Lilja M, Savolainen MJ, Reunanen A. Prevalence of the metabolic syndrome in drug-treated hypertensive patients and control subjects. J Intern Med. 1999;245:163-174.

37. Organización Panamericana de la Salud. HEARTS: Paquete técnico para el manejo de las enfermedades cardiovasculares en la atención primaria de salud. Washington, DC: OPS, 2019.

38. Williams B, Mancia G, Spiering W, et al. 2018 ESC/ESH Guidelines for the management of arterial hypertension. Eur Heart J. 2018;39(33):3021-3104.

39. Leung AA, Nerenberg K, Daskalopoulou SS, et al. Hypertension Canada's 2016 Canadian Hypertension Education Program Guidelines for Blood Pressure Measurement, Diagnosis, Assessment of Risk, Prevention, and Treatment of Hypertension. Can J Cardiol. 2016;32(5):569-588.

40. Wright Jr JT, Fine Lj, Lackland DT, Ogedegbe G, Dennison Himmelfarb CR. Evidence Supporting a Systolic Blood Pressure Goal of Less Than $150 \mathrm{~mm} \mathrm{Hg}$ in Patients Aged 60 Years or Older: The Minority View. Ann Intern Med. 2014;1(160):7-499.

41. Tsujimoto T, Kajio H. Benefits of Intensive Blood Pressure Treatment in Patients With Type 2 Diabetes Mellitus Receiving Standard but Not Intensive Glycemic Control. Hypertension. 2018;72(2): 323-330.

42. Campbell NR, Lackland DT, Niebylski ML, Liga Mundial de la Hipertensión, Comité Ejecutivo de la Sociedad Internacional para la Hipertensión. High Blood Pressure: Why Prevention and Control are Urgent and Important-A 2014 Fact Sheet From the World 
Hypertension League and the International Society of Hypertension. J Clin Hypertens. 2014;16(8):551-553.

43. Campbell NR, Khalsa T, Lackland DT, et al. High Blood Pressure 2016: Why Prevention and Control Are Urgent and Important. The World Hypertension League, International Society of Hypertension, World Stroke Organization, International Diabetes Foundation, International Council of Cardiovascular Prevention and Rehabilitation, International Society of Nephrology. J Clin Hypertens (Greenwich). 2016;18(8):714-717.

44. Bennett JE, Stevens GA, Mathers CD, et al. NCD Countdown 2030: worldwide trends in non-communicable disease mortality and progress towards Sustainable Development Goal target 3.4. Lancet. 2018;392(10152):1072-1088.

45. Kotchen TA. The search for strategies to control hypertension. Circulation. 2010;122(12):1141-1143.

46. Organización Panamericana de la Salud. HEARTS: Paquete técnico para el manejo de las enfermedades cardiovasculares en la atención primaria de salud: Evidencia: protocolos de tratamiento clínico basados en la evidencia. Washington, DC: OPS; 2019.

47. Organización Panamericana de la Salud. HEARTS: Paquete técnico para el manejo de las enfermedades cardiovasculares en la atención primaria de salud: Acceso a medicamentos y tecnologías esenciales. Washington, DC: OPS, 2019

48. Organización Panamericana de la Salud. HEARTS: Paquete técnico para el manejo de las enfermedades cardiovasculares en la atención primaria de salud: Hábitos y estilos de vida saludables: asesoramiento para los pacientes. Washington, DC: OPS; 2019.

49. Organización Panamericana de la Salud. HEARTS: Paquete técnico para el manejo de las enfermedades cardiovasculares en la atención primaria de salud: Sistemas de monitoreo. Washington, DC: OPS; 2019. Disponible en https://www.paho.org/hq/index. php?option $=$ com_content\&view $=$ article\&id $=15053$ :hearts-technical-package\&Itemid $=3465 \&$ lang $=$ es.

50. Organización Panamericana de la Salud. HEARTS: Paquete técnico para el manejo de las enfermedades cardiovasculares en la atención primaria de salud: Trabajo basado en equipos multidisciplinarios. Washington, DC: OPS; 2019.

51. Frieden TR, Jaffe MG. Saving 100 million lives by improving global treatment of hypertension and reducing cardiovascular disease risk factors. J Clin Hypertens (Greenwich). 2018;20:208-211.

52. Kaczorowski J, Chambers LW, Dolovich L, et al. mproving cardiovascular health at population level: 39 community cluster randomised trial of Cardiovascular Health Awareness Program (CHAP). BMJ. 2011;342:d442.

53. Ferrari P. Reasons for therapeutic inertia when managing hypertension in clinical practice in non-Western countries. J Hum Hypertens. 2008;DOI:10.1038/jhh.2008.1 17.

54. Okonofua EC, Simpson KN, Jesri A, Rehman SU, Durkalski VL, Egan BM. T Therapeutic Inertia Is an Impediment to Achieving the Healthy People 2010 Blood Pressure Control Goals. Hypertension. 2006;47(3):345-351.

55. Mangat BK, Campbell N, Mohan S, et al. Resources for Blood Pressure Screening Programs in Low Resource Settings: A Guide From the World Hypertension League. J Clin Hypertens (Greenwich). 2015;17(6):418-420.

56. Campbell N, Touyz R, Lackland D, Redburn K, Niebylski M. Celebrate World Hypertension Day (WHD) on May 17, 2015, and Contribute to Improving Awareness of Hypertension. J Clin Hypertens (Greenwich). 2015;17(4):317-318.

57. Campbell NC, Lackland DT, Lisheng L, et al. L The World Hypertension League: A Look Back and a Vision Forward. J Clin Hypertens (Greenwich). 2015;17(1):5-6.

58. Thomopoulos C, Parati G, Zanchetti A. Effects of blood pressure lowering treatment in hypertension: 8 . Outcome reductions vs. discontinuations because of adverse drug events - meta-analyses of randomized trials. J Hypertens. 2016;34(8):1451-1463.

59. Organización Panamericana de la Salud. HEARTS: Paquete técnico para el manejo de las enfermedades cardiovasculares en la atención primaria de salud. Washington, DC: OPS; 2018.

60. Mills KT, Obst KM, Shen W, et al. Comparative Effectiveness of Implementation Strategies for Blood Pressure Control in Hypertensive Patients: A Systematic Review and Meta-analysis. Ann Intern Med. 2018;168(2):110-120.
61. Frieden TR, King SM, Wright JS. Protocol-based treatment of hypertension: a critical step on the pathway to progress. JAMA. 2014;311(1):21-22.

62. Organización Panamericana de la Salud. HEARTS: Paquete técnico para el manejo de las enfermedades cardiovasculares en la atención primaria de salud: Guía de implementación. Washington, DC: OPS; 2018.

63. Campbell N, Tobe S. The Canadian effort to prevent and control hypertension. Can other countries adopt Canadian strategies? Curr Opin Cardiol. 2010;25(4):366-372.

64. Parati G, Stergiou GS, Asmar R, et al. European Society of Hypertension Practice Guidelines for home blood pressure monitoring. J Hum Hypertens. 2010:1-7.

65. Parati G, Omboni S, Albini F, et al. Home blood pressure telemonitoring improves hypertension control in general practice. The TeleBPCare study. J Hypertens. 2009;27(1):198-203.

66. Albini F, Xiaoqiu L, Torlasco C, et al. An ICT and mobile health integrated approach to optimize patients' education on hypertension and its management by physicians: The Patients Optimal Strategy of Treatment(POST) pilot study. Actas de conferencia: Conferencia anual de la Sociedad de Ingeniería en Medicina y Biología (IEEE). 2016;2016:517-520.

67. Widmer RJ, Collins NM, Collins CS, West CP, Lerman LO, Lerman A. Digital Health Interventions for the Prevention of Cardiovascular Disease: A Systematic Review and Meta-analysis. Mayo Clin Proc. 2015;90(4):469-480.

68. Parati G, Stergiou GS, Asmar R, et al. European Society of Hypertension guidelines for blood pressure monitoring at home: a summary report of the Second International Consensus Conference on Home Blood Pressure Monitoring. J Hypertens. 2008;26(8):1505-1526.

69. Burke LE, Ma J, Azar KM, et al. Current Science on Consumer Use of Mobile Health for Cardiovascular Disease Prevention: A Scientific Statement From the American Heart Association. Circulation. 2015;132(12):1157-1213.

70. Campbell NR, Berbari AE, Cloutier L, et al. Policy Statement of the World Hypertension League on Noninvasive Blood Pressure Measurement Devices and Blood Pressure Measurement in the Clinical or Community Setting. J Clin Hypertens (Greenwich). 2014;16(5):320-322.

71. Campbell NR, Gelfer M, Stergiou GS, et al. A Call to Regulate Manufacture and Marketing of Blood Pressure Devices and Cuffs. A Position Statement From the World Hypertension League, International Society of Hypertension and Supporting Hypertension Organizations. J Clin Hypertens. 2016;18(5):378-380.

72. Padwal R, Campbell NRC, Schutte AE, et al. Optimizing observer performance of clinic blood pressure measurement: a position statement from the Lancet Commission on Hypertension Group. J Hypertens. 2019; DOI:10.1097/HJH.0000000000002112.

73. Campbell N, Ordúnez P, Jaffe MG, et al. Implementing standardized performance indicators to improve hypertension control at both the population and healthcare organization levels. J Clin Hypertens (Greenwich). 2017;19(5):456-461.

74. Campbell NRC, Ordúnez P, DiPette DJ, et al. Monitoring and evaluation framework for hypertension programs. A collaboration between the Pan American Health Organization and World Hypertension League. J Clin Hypertens (Greenwich). 2018;DOI:10.111/JCH.1330 7.

75. Campbell NR, McAlister FA, Quan H. Monitoring and Evaluating Efforts to Control Hypertension in Canada: Why, How, and What It Tells Us Needs to Be Done About Current Care Gaps. Can J Cardiol. 2013;29:564-570.

76. Benjamin EJ, Muntner P, Alonso A, et al. Heart Disease and Stroke Statistics-2019 Update: A Report From the American Heart Association. Circulation. 2019;139(10):e56-e66.

77. Owolabi MO, Sarfo F, Akinyemi R, et al. Dominant modifiable risk factors for stroke in Ghana and Nigeria (SIREN): a case-control study. Lancet Glob Health. 2018;6(4):e436-e446.

Manuscrito original en inglés recibido el 25 de septiembre de 2019, aceptado el 5 de noviembre de 2019 . 


\section{São Paulo call to action for the prevention and control of high blood pressure: 2020}

ABSTRACT - About 1/4th of adults have high blood pressure which is the single most important risk for death (including heart disease and stroke).

- There are effective policies that could facilitate people making healthy choices to prevent raised blood pressure, and if fully implemented, could largely prevent hypertension from occurring.

- Hypertension is easy to screen and treat for BUT only about $50 \%$ of adults with hypertension are aware of their condition and only about 1 in 7 is adequately treated.

- Preventing and controlling high blood pressure is the major mechanism for NCD prevention and control and a model for other NCD risks.

- Effective lifestyle and drug treatments could prevent and control hypertension in most individuals if systematically applied to the population, simple interventions are feasible in all settings, and can be used to enhance primary care.

- Urgent sustained action is needed is needed for effective public policies and health system changes to prevent and control hypertension.

Keywords Hypertension; cardiovascular diseases; global health; diet, sodium-restricted; stroke.

\section{Chamado à ação de São Paulo para prevenção e controle da hipertensão arterial: 2020}

RESUMO

- Cerca de $1 / 4$ dos adultos têm hipertensão arterial, que é o fator de risco isolado mais importante para morte (incluídas as mortes por cardiopatia e acidente vascular cerebral).

- Existem políticas eficazes que poderiam facilitar escolhas pessoais saudáveis para evitar a elevação da pressão arterial e, se plenamente implementadas, podem prevenir a ocorrência da hipertensão arterial.

- É fácil rastrear e tratar a hipertensão, MAS somente cerca de 50\% dos adultos hipertensos estão cientes de sua condição, e apenas cerca de 1 em cada 7 é tratado adequadamente.

- A prevenção e controle da hipertensão é o principal mecanismo de prevenção e controle das doenças não transmissíveis e um modelo para outros riscos de doenças não transmissíveis.

- Tratamentos eficazes com mudanças de estilo de vida e medicamentos poderiam prevenir e controlar a hipertensão arterial na maioria das pessoas se aplicados sistematicamente à população; as intervenções simples são viáveis em todos os ambientes e podem melhorar a atenção primária.

- É necessária a ação continuada e urgente a fim de obter mudanças efetivas nas políticas públicas e no sistema de saúde para prevenir e controlar a hipertensão arterial.

Palavras-chave Hipertensão; doenças cardiovasculares; saúde global; dieta hipossódica; acidente vascular cerebral. 